Again, the same point is illustrated by the graphs of two rats of the same weight-namely, 113 grams-both inoculated with $T$. gambiense.

Rat 1 (animal house), had an average number of 54,171 trypanosomes per c.mm. of blood per day during the first ten days of infection.

Rat 2 (cold chamber), had an average number of 14,728 trypanosomes per c.mm. per day during the first ten days of infection.

We must admit, however, the existence of cases which did not seem to react to the cold, and in which the parasites quickly developed, in spite of treatment; but the conclusion to be drawn from all our animals is that the parasites tend to be fewer in the cold if the animal is not sabjected to exposure. This we would explain by the fact that the body resistance of the animal is raised.

I would also point out that the charts refer to two selected cases, chosen because the animals were much the same in weight. We must be guarded in drawing hasty conclusions till further work has been done and until the question of individual resistance of the animal hosts (apart from treatment) has been further considered. Thesechart show distinctly lower numbers of parasites in those treated in the cold chamber.

We found that in rats the incubation period of $T$. bruce was delayed in the cold, but the parasites develop very rapidly afterwards, and the animals are quickly killed (in about six days). In guinea-pigs we found that the incu. bation period was three days in both the cold and the animal house, but the animal kept in the cold developed the trypanosomes much more slowly. In four rats inoculated with $\boldsymbol{S} p$. duttoni we found that the parasites were less numerous in the peripheral blood of those in the cold chamber.

Summary.

1. We must distinguish carefully between cold and exposure, only the former being likely to be beneficial.

2. Animals in the cold chamber were livelier than those in the animal house, and the fact that the majority of these gained weight shows a favourable intluence on nutrition.

3. Experiments in rats and guinea-pigs inoculated with $T$. gambiense and $T$. rhodesiense show :

(a) Delayed incubation in the cold.

(b) Prolongation of life in the cold.

(c) Tendency for fewer parasites to occur in the peripheral blood of animals kept in the cold.

4. Incubation period of $T$. lewisi and $T$.brucei delayed in the cold.

5. Prolongation of life of animals infected with Caderas and Nagana.

6. It was noted in two guinea-pigs after inoculation with tabercle that the superficial abscess which formed at the site of inoculation developed much more slowly in the cold (a month later).

7. In rats infected with Spirochaeta duttoni the numbers of parasites circulating in the peripheral blood were fewer in the animals treated in the cold chamber, and this was also observed in guines-pigs infected with $T$. brucei.

8. The patient, W.A., said he felt better after his sojourn $n$ the cold chamber.

1906. W. W. Crane, M. D.: Cold Air in the Treatment of Tetanus and

Yellow Fever, St. Louis Medical Review, July 7th, 1906. à l'hibernation. Extrait des Archives de parasitologie, tome $\mathrm{xi}_{\text {, }}$ No. 3,1907 .

1909. Dr. Luis Perna: The Treatment of Yellow Fever, Sanidad Beneficencia August, 1909 .

1910. C. C. Bass : Climatic Treatment of Pellagra, preliminary note Journ. Amer. Med. As80c., September 10th, 1910

1910. Mrior R. Ross and Major C. I. Williams : Preliminary Trperiments on the Effect of Cold on Various Diseases in Speril Animals. Annals of Tropical Medicine and Parasitology, vol. iv, No. 2, July. 1910.

1910. Raubitschek (Hugo): Wien. klin. Woch., June 30th, 1910, xxiii, 6 Zur pathogenese der Pellagra, pp. 963-5.

1910. R Ross: Prevention of Malaria. London: John Murray.

1911. Major R. Ross and J. G. Thomson: Proc. Roy. Soc., B, vol. Ixxxiii,

UNDER the will of the late Mr. William Taylor of Eastbourne, the Consumption Hospital, the Cancer Hospital, the Royal Hospital for Incurables, Putney; the Royal Free Hospital, London; the Royal Infirmary, Liverpool and the Home for Confirmed Invalids each receive (subject to the life interest of the testator's wife) a sum of $£ 2,000$, half that sum going to the Samaritan Free Hospital for Women.

\section{AN ANALYSIS OF \\ THREE HUNDRED AND TWENTY-SIX CASES OF ASIATIC CHOLERA.}

\author{
BY HENRY STEVENS, M.R.C.S., L.R.C.P., \\ FUNCHAL, MADEIRA.
}

I AM indebted to my friend Dr. Lomelino for the following statistics. He was medical officer in charge of the Funchal Isolation Hospital during the recent cholera epidemic, and I had an opportunity of watching his devoted work during that period.

From the time that he took over the charge of the isolation hospital until the end of the epidemic he had under his care 326 cases of cholera, of which 120 ended fatally.

Deaths.
3 died of syncope during convalescence, but had previous history of morbus cordis.

2 died of meningitis.

1 died of bronchopneumonis.

1 died of thrombosis and pulmonary embolism.

2 died of uraemic coma following upon suppression of urine.

2 arrived dead.

5 arrived moribund and died within two hours.

The remainder died of collapse while under treatment.

Of the 326 cases, in roughly 75 per cent. there was an acute onset; in many it was fulminating. In the remaining 25 per cent. the onset was gradual. The duration of symptoms in those who recovered was three to ten days. In only 5 per cent. of the cases were cramps in the legs and abdomen absent. After acute symptoms had passed off some 3 per cent. of the patients went into a condition resembling typhoid, with tympanites, slight secondary diarrhoea, headache, and temperature above $101^{\circ}$ for more than a week. Some of these gave Widal's reaction in the stronger dilutions, but they all recovered within a fortnight.

About 10 per cent. of the patients were chronic alcoholics, and this class of the community seemed especially prone to take the disease.

Of the female patients fully 10 per cent. were lactating which seems a high percentage. There were also 5 pregnant women among the patients. Of these, 4 aborted, 2 between the second and third month and 2 between the sixth and serenth month; 2 died, but without any puerperal complications.

Among the 326 patients there were 30 babies under 3 years of age and 42 persons over 60 .

\section{Complications.}

Among the 326 cases there were :

$$
\begin{aligned}
& \text { Annria of more than three days Cases. } \\
& \text { Anuria of more than three days } \quad \ldots \quad 15 \text { (only } 2 \text { died) }
\end{aligned}
$$

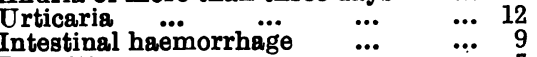

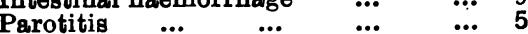

$$
\begin{aligned}
& \begin{array}{lllll}
\text { Parotitis } & \ldots & & & \\
\text { Thrombosis and embolism } & \ldots & \ldots & 5 \\
\text { (fatal) }
\end{array} \\
& \text { Temperature of over } 101^{\circ} \text { for more } \\
& \text { than first week of convalescence ... } 8 \\
& \text { Ophthalmic complications : }
\end{aligned}
$$

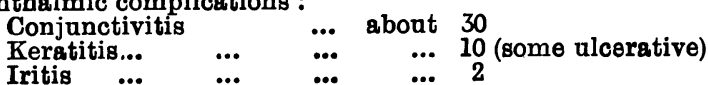

The epidemic, comprising about 1,700 cases for the whole island, has been disseminated entirely by food and actual contact, and not by means of water supply. It has only been stemped out by rigid isolation or segregation of all "contrats."

Systematic examination of the faeces was carried out in the case of all convalescents, all inmates of the "contacts" segregation block, and all hospital em. ployees. Of the 206 convalescents, 20 carried the vibrio in the faeces for more than a fortnight after the disappearance of all active symptoms. Out of about 350 inmates of the "contacts" "block, only 6 actually developed the disesse, but many who never showed sym. ptoms were found to carry the vibrio in their faeces for more than three weeks after being in contact with a patient. 
Out of 71 people employed in various capacities at this hospital, in no single instance was it possible to show the presence of the vibrio in the faeces. This speaks well for the excellent precantions adopted by the anthorities.

The fact that in nearly all cases which have been carefully investigated it has been possible to establish a history of direct contact, or at any rate a visit to an infected house, has gone far towards establishing the limits of the incubation period. These seem to be from a few hours up to eight or nine days. A man in the poor quarter of Funchal, out of bravado, wrapped himself in the blankets of a cholera patient. He developed the disease in six hours, and died in eighteen hours. His family of three all developed the disease subsequently, but not until six days after his death.

The severe outbreak at the neighbouring township of Machico was traceable to one man who had slept a night in an infected house in Funchal, and developed the disease three days after his return.

Of the "contacts" who were isolated in their own homes, instead of being segregated in the isolstion hospitals, a very much larger proportion than the abovementioned 6 out of 350 subsequently developed cholera. This seems to lend colour to the statement one often sees, that even when carrying the germ one can probably avoid the disease by regular meals and by careful hygiene, such as was observed in the "contacts" block.

\section{Clinical Notes.}

The probable explanation of the frequency of ophthalmic complications lies in the fact that the secretion of tears is almost entirely inhibited, as is the case with all the other fluid secretions of the body, owing to the tremendous loss of fluid by diarrhoea. From the same cause stomatitis, parotitis, laryngitis, and bronchitis were of relatively frequent occurrence.

A striking featare of many cases, which I have not seen mentioned in the textbooks, was the continuance of watery, choleraic vomiting long after the diarrhoea had stopped. In the only case I had to treat among the foreign residents this occurred, the vomiting continuing for fortyeight hours after the diarrhoea had ceased.

The fewness of the cases of prolonged anuria in this epidemic was probably due to the use of saline infusion as a routine treatment in all severe cases. Urticaria was just as common among the mild cases as among the severe, where saline infusion had been resorted to.

The disease has been almost entirely limited to the lowest and dirtiest of the population. No English residents have had it.

Pathological Notes.

Autopsies were only carried out in a few cases, owing to the tremendous prejudice of the peasents. In these few instances it was found that there had been great loss of the gastric mucosa, though not to quite the same extent as with the mucous lining of the jejunum and ileum, where the mucosa had been so completely cast off that it is difficult to understand how regeneration can ever take place. In most cases the bacteriological examination of the faeces and vomit seemed to show that the comma vibrio remains in undisputed possession of the alimentary canal for the whole period of the disease, to the exclusion of all the ordinary intestinal flora. Those cases in which the patient subsequently fell into a sort of typhoid state were probably instances of the comma bacillas failing to establish this undisputed possession, so that there was a rapid absorp. tion of intestinal toxins through the denuded mucose, resulting in a condition of profound copraemia. In these cases, and also in the afebrile secondary diarrhoeas, very good results were obtained with intestinal irrigation and the exhibition of such drugs as salol and benzo-naphthol There were one or two cases of long-standing enteritis (a common condition among the poor here) in which a complete cure was the result of a sharp attack of cholera. In one case, a woman aged 74, who had been the victim of mucous enteritis for ten years, and had been bedridden for two years, left the hospital after an attack of cholera with complete cessation of all symptoms of her former malady

I can cordially endorse the remarks of my colleague, Dr. Scott, in his letter to the British Medical Journar of
February 18th, in which he attributes the prompt stamping out of this epidemic to the excellent measures prescribed by Drs. Magalhaes and Carlos Franca, who were sent from Lisbon. I must again thank Dr. Lomelino for his great kindness in placing this material at my disposal and for the opportunities which he has given us English doctors of seeing the cases under his care.

\section{THE AFTER-HISTORY OF THIRTY CASES:OF MOVABLE KIDNEY TREATED BY OPERATION.}

BY

A. RENDLE SHORT, B.Sc., M.D., B.S., F.R.C.S. HONORARY SURGICAL REGISTRAR, BRISTOL ROYAL INFIRMARY; DEMONBTRATOR OF PHYSIOLOGY, UNIVERSITY OF BRISTOL.

Mors than one writer on surgical treatment has complained that therie are in the literature fow reliable statistics of the end-results of the various forms of opera. tive treatment practised for movable kidney. There are a few figures culled from the private practice of individual surgeons, but these always need supplementing by hospital reports before they can be used as indications for treat. ment by other hands. The writer claims to be unbiassed in favour of any particular operation.

Appended is a list of all the cases of movable kidney treated by operation during the ten years 1900 to 1909 at the Bristol Royal Infirmary It is possible that one or two may have been accidentally omitted. The after.history has been obtained, occasionally by finding a note of a subsequent visit to the infirmary, occasionally by report of the medical attendant, but in most cases by correspondence. Many of the patients kindly came to be personally interviowed by me. They were examined both sitting up and lying down.

Unless otherwise stated, the indication for the operation. was repeated attacks of dragging pain. It is not castomary to operate here unless the kidney is sufficiently movable to allow the hands to get above and hold it down.

I have thought it better to include in the list even those whose after-history could not be traced, owing to removal from residence, etc. The total number reported on is therefore 38, which, however, represents more than that number of patients, on account of relapses on the same or opposite side of the body. Daring the same period, 21,143 surgical patients were treated at the infirmary.

It may be remarked that all the patients were women, that the ages ranged from 22 to 58, and that the right kidney alone was operated on in every case except one (E. D.), in whom both sides were fixed at an interval of a year.

It has not been customary to prescribe a belt after operation.

Operations Performed.

Three totally distinct operations are well represented. The first shows a good deal of variety in detail.

1. Transcortical Suturing.-The kidney is stripped of its fatty capsule, and as far as possible of its true fibrous capsule. Sutures, usually three, are passed by means of a curved needle through the substance of the organ, and by them it is fixed to the muscles of the loin, taking a deep hold both of kidney and of muscles. In at least three cases (probably more), a turn including the last rib was taken. The suture material has usually been stout catgut, in some cases reindeer tendon, and occasionally silk or silkworm gut. The muscles are then carefully sewn together. An effort has usually been made to avoid injuring the last dorsal nerve. I have incladed here two cuses in which mattress sutures were carried through capsule and kidney substance, muscles and skin.

2. Fixation by the Capsule only.-The true fibrous capsule is partially stripped off, and satures of catgut or reindeer tendon are used to attach the flaps of capsule to the muscles.

3. Pure Carbolic Acid and an Iodoform Gauze Sling.The kidney is exposed by the usual lumbar incision, the fatty capsule removed, and the whole surface painted with pare carbolic acid. Then a sling of iodoform ganze is arranged to support the lower pole, the ends being brought 